\title{
GTL or LNG: Which is the best way to monetize "stranded" natural gas?
}

\author{
Dong Lichun, Wei Shun'an, Tan Shiyu* and Zhang Hongjing
}

School of Chemistry and Chemical Engineering, Chongqing University, Chongqing 400044, China

\begin{abstract}
A large portion of world's natural gas reserves are "stranded" resources, the drive to monetize these resources leads to the development of gas-to-liquids (GTL) and liquefied natural gas (LNG) technologies. LNG has the advantage of having been developed for the past 40 years and having an excellent safety record. GTL on the other hand is another option with substantial benefits, but its development stage and commercial viability are far behind LNG. This paper presents a techno-economic comparison of GTL with LNG, including technical development, plant efficiency, market potential for the products, and capital cost for the infrastructure. The aim is to give an overall view on both LNG and GTL and provide a perspective on the profitability of these two technologies.
\end{abstract}

Key words: GTL (gas to liquids), LNG (liquefied natural gas), “stranded” natural gas

\section{Introduction}

Natural gas is playing a more and more important role in the supply of energy for both industrial and domestic use. The total global annual gas consumption is forecast to rise to 4.59 trillion cubic meters by 2020 with an annual increase rate of $3.2 \%$ (Chen, 2003), which is much faster than that of oil and coal. The world's plentiful gas supply sources, the desire for less carbon-intensive fuels, and the need for cleaner air are driving a continuous innovation of gas technologies. However, a considerable portion of the world natural gas reserves fall into the category termed as "stranded", which means that the gas reserves are either located remotely from consumers or are sporadic in the region where any single reserve is relatively small. For the "stranded" gas, the conventional means of transportation via pipeline is usually not practical or economical because of geographical, political, or diplomatic limitations. The owners of the "stranded" gas face a challenge on how to monetize the large stranded gas resources. This drive leads to the developments in LNG and GTL technologies. The LNG is essentially a physical process converting natural gas to liquid for easy transportation, while GTL is a chemical process that methane is converted to naphtha, transportation fuels, and specialty chemicals via Fischer-Tropsch (F-T) synthesis or into methanol and into gasoline with a methanol to gasoline (MTG) direct process. The discussion in this paper is focused on Fischer-Tropsch synthesis. The LNG and GTL products offer environmental benefits over other conventional fuels such as coal and products derived from crude oil; both offer excellent prospects to countries having gas resources with potential for monetization.

*Corresponding author. email: tshy@cqu.edu.cn Received July 23, 2008
LNG and GTL serve different energy markets with different marketing systems, policies and strategies. The comparison between LNG and GTL is the most prominent debate for resource owners, developers and investors alike. Several factors have to be considered to evaluate the project economics. LNG has the obvious advantage of having been developed for the past 40 years and has to-date enjoyed robust growth and has an excellent safety record. GTL on the other hand is a developing alternative to LNG with substantial benefits in terms of sustainable economic, social and environmental development. With the recent development of GTL technology, the debate between GTL and LNG is no longer "is the project economic" but rather "which project is most profitable". This paper examines the technical performance and market potential for LNG and GTL products, and the capital costs for LNG and GTL infrastructure. The aim is to give an overall view on both LNG and GTL and provide a perspective on the profitability of the two technologies (Pyrdol and Baron, 2006).

\section{Technical developments of GTL and LNG}

The LNG process first involves a gas treatment plant for removal of acid gas (sulfur, carbon dioxide), water, and other contaminants. The gas is then cooled to separate the heavier hydrocarbons such as $\mathrm{C}_{3}, \mathrm{C}_{4}$, and $\mathrm{C}_{5+}$ components. These heavier components are then fractionated to produce $\mathrm{C}_{5+}$ and Liquefied petroleum gas (LPG) products. The purified gas is then liquefied in cryogenic exchangers at a temperature of $-162{ }^{\circ} \mathrm{C}$. LNG, occupies only $1 / 600$ of its original volume in the gaseous state, is then stored in LNG tanks prior to shipping to market in heavily insulated tankers and regasified for use in conventional gas markets such as power generation and domestic applications (Yang and Wang, 2005). A typical LNG process is as Fig. 1.

Like the LNG process, the GTL process also starts with 


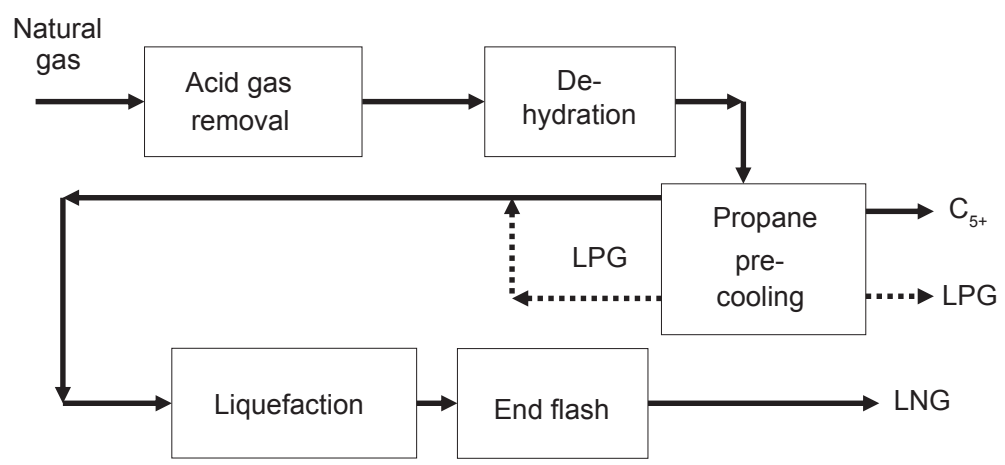

Fig. 1 The schema of a typical LNG process

a gas plant for removal of sulfur, carbon dioxide, water, other contaminants and heavier hydrocarbon components. However, unlike the LNG process, which is a simple physical process to liquefy natural gas at a cryogenic temperature, the GTL process involves several complex chemical reactions. A GTL unit comprises of three core technologies: synthesis gas (syngas) manufacture, Fischer-Tropsch (F-T) synthesis and hydrocracking (Hu et al, 2006; Fleisch et al, 2002; Heng and Idrus, 2004; Bakkerud, 2005; Sie, 1998).

In the syngas manufacture process, the purified gas is converted to syngas by partial oxidation, steam reforming, or a combination of the two processes. The syngas is predetermined with a mixture of hydrogen and carbon monoxide with a 2:1 ratio of hydrogen to carbon monoxide as the feedstock of Fischer-Tropsch synthesis. The syngas is then converted to paraffinic hydrocarbons in a F-T Reactor with the use of cobalt or iron based catalyst. This stage is the key to the commercial success of the GTL process, and high yields of desirable middle distillate products are essential to lower unit cost. In the final stage of the GTL process, the raw F-T hydrocarbons are subsequently upgraded to final products by using conventional refinery processes: wax hydrocracking, distillate hydrotreating, catalytic reforming, etc. The primary products include naphtha, and transportation fuels such as diesel and jet fuels. GTL is also an efficient process for producing high quality lubes, waxes and white oils, which are utilized in the food and pharmaceutical industry. A simplified GTL-F-T process is shown in Fig. 2.

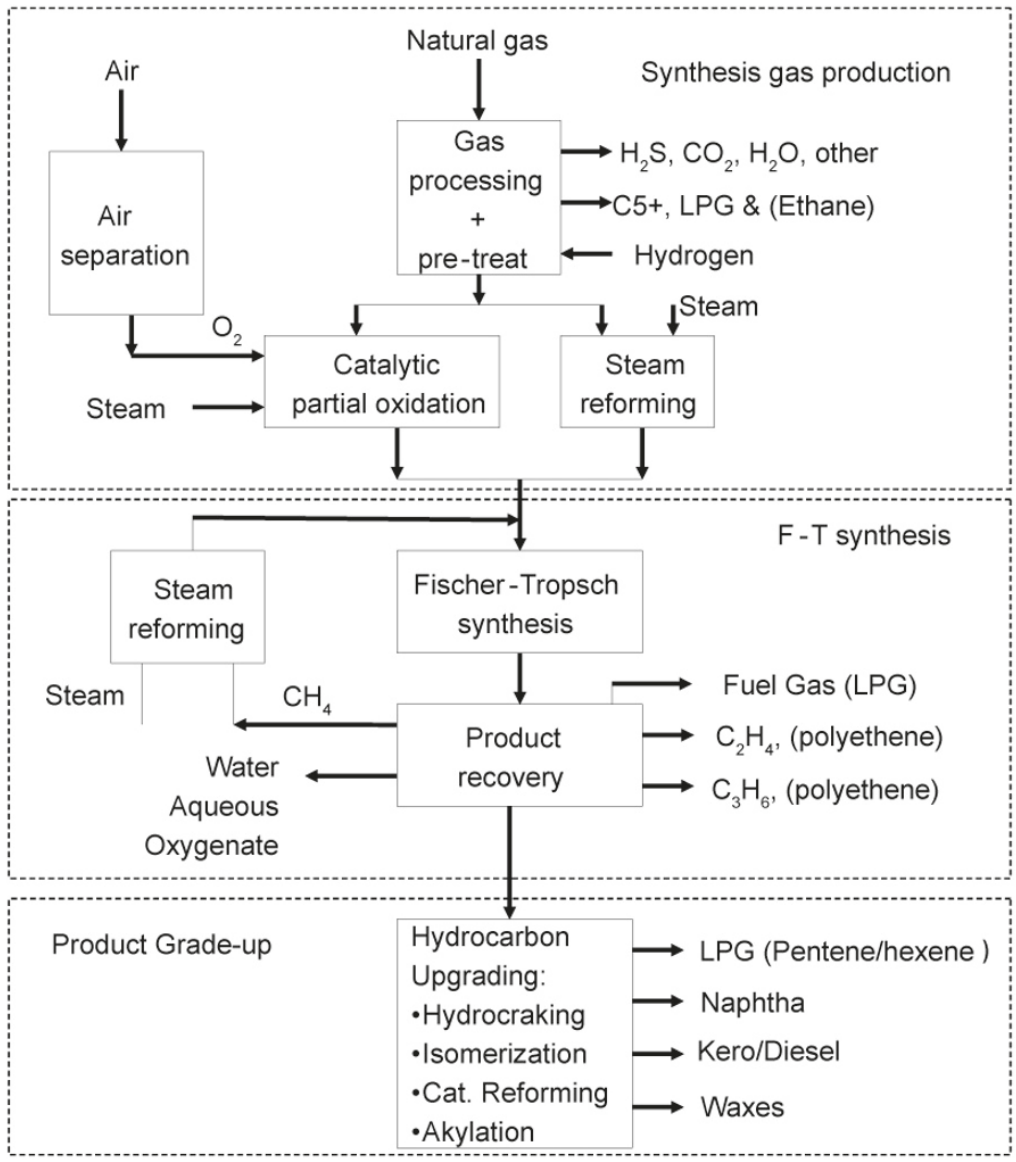

Fig. 2 The overall schema of a GTL process 
Although long achieving technical success, GTL has not been economically competitive, and LNG has been actually the only commercial option for the owners of "stranded" gas, until recently. Today we see a resurrecting second generation GTL process using low-temperature F-T conversion as a result of abundant gas supply and strong need of highquality transportation fuels. The increasing efficiency of the GTL process and the ability to build bigger plants of commercial scale based on operational experience make GTL an attractive alternative to LNG for the gas owners to monetize the "stranded" gas resources. However, the GTL process is still in its infancy, and only Shell SMDS and Sasol Synthol are in commercial operation. The other processes, such as Rentech, ExxonMobil AGC-21 and Syntroleum are still in the demonstration stage. Therefore, GTL production has significantly larger technical risk than LNG production at present.

\section{Plant efficiency of GTL and LNG facilities}

Two parameters are often used to define the efficiency of LNG and GTL facilities. The first is Thermal (Energy) Efficiency (TE), which is defined as

$$
\mathrm{TE}=\frac{\text { LHV of liquid final products }}{\text { LHV of natural } \mathrm{g} \text { as }} \times 100 \%
$$

where LHV stands for lower heating value. The other is
Carbon Efficiency (CE), which is defined as

$$
\mathrm{CE}=\frac{\text { Carbon molecules in the final products }}{\text { Carbon molecules in natural gas }} \times 100 \%
$$

Thermal Efficiency is a measure as how much the total energy in the feedstock is utilized to produce the final hydrocarbon product, whereas Carbon Efficiency is essentially a measure as how best the carbon atoms in the feedstock are utilized to produce the final product.

The Thermal Efficiency of GTL is considered low and is typically around $60 \%$; LNG on the other hand has a Thermal Efficiency around $88 \%$. The Carbon Efficiency of the GTL process is around $77 \%$ with the remainder of the carbon being converted to $\mathrm{CO}_{2}$. LNG production on the other hand has a Carbon Efficiency of around $88 \%$. The efficiency of GTL technology is relatively low compared with LNG technology. Advances in the GTL technology are projected to increase the carbon efficiency and the thermal efficiency to $90 \%$ and $73 \%$ respectively within the next 10 years (Pyrdol and Baron, 2006; Fleisch et al, 2002; Smith, 2004; Patel, 2005). A typical energy and carbon balance of GTL is shown in Fig. 3 .

LNG has higher processing efficiency, then LNG can deliver more energy to the market than GTL for a given quantity of natural gas feed, however, the GTL products are more valuable, one can not declare that LNG is the ultimate winner simply from the delivered energy.

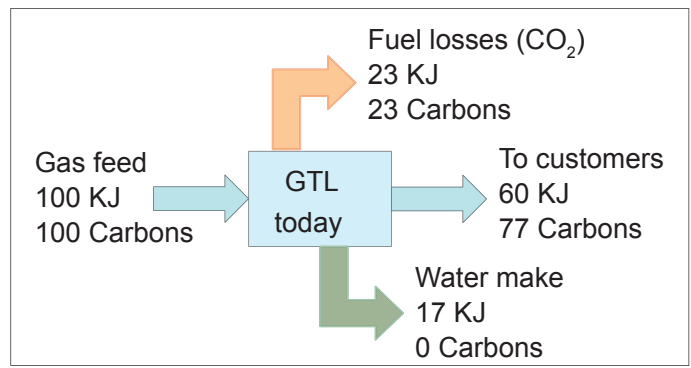

Energy Efficiency: 60\% (at present) Carbon Efficiency: 77\%

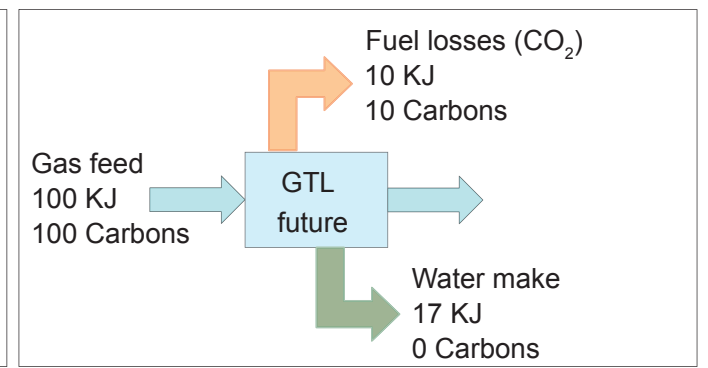

$73 \%$ (after 10 years) $90 \%$

Fig. 3 The plant efficiency of GTL

\section{Products and market}

The primary market for LNG is power generation, industrial fuel and domestic and commercial heating and air-conditioning. Since its inauguration in 1964, LNG has consistently increased its share in world's natural gas trade. The total LNG trade is $8 \times 10^{4}$ tonnes in 1964 and increased to $1.318 \times 10^{8}$ tonnes in 2004 with an annual increase rate of $20.34 \%$. If we just take the development from 1994 to 2004 into account, the annual increase rate is also as high as $7.31 \%$. The portion of LNG trade in the world total natural gas trade increased from $0.3 \%$ in 1970 to $26.2 \%$ in 2004 . According to
BCC (Business Communication Company, USA), the total LNG trade will reach $2.50 \times 10^{9}$ tonnes in 2010 (Zeng, 2006).

Before 2000, the world LNG trade was in a period of short-term balance. However, with the strong rise in the price of crude oil from the winter of 2000 , the world LNG demand has grown rapidly and the demand of LNG exceeded the supply from 2004. As the three major markets of LNG, the import of Asia, European, and North America was 9.23× $10^{7} \mathrm{t}, 3.74 \times 10^{7} \mathrm{t}$, and $1.36 \times 10^{7} \mathrm{t}$ in 2005 , and was increased by $9.6 \%, 25.9 \%$, and $21.4 \%$ compared to that in 2004 , respectively. This supply/demand imbalance combined with $\mathrm{w}$ high oil price caused a significant rise of LNG price for 
long-term contracts and on the open market. The FOB price of LNG contract signed in 2003 was less than \$3.5/MMBTU $\left(1 \mathrm{MMBTU}=28 \mathrm{~m}^{3}\right)$, whereas the price increased to $\$ 5 /$ MMBTU at the end of 2005, and \$6/MMBTU in 2006 (Zeng, 2006; Zhang and Pang, 2005; Wang, 2005).

Due to the large growth potential in the LNG market, the supply-demand balance for LNG in the short to medium term is forecasted to remain very competitive. However, the world natural gas resource is abundant and the high price of LNG has attracted aggressive investment in LNG facilities, which is estimated to be over $\$ 67$ billion from 2005 to 2009, and the potential supply of LNG will exceed demand. The imbalance situation of LNG supply versus demand is forecasted to change after 2010 and the prices of LNG would possibly decline in a long-term prospect. Therefore, many natural gas owners are devoting a lot of effort to develop other competitive projects except LNG facilities and gain market share even while the LNG prices remain fairly aggressive.

The pricing mechanism for LNG is usually based on long-term commitment by the supplier and consumer. Via long-term contract, the suppliers can reduce the high risk for building new LNG facilities and the buyers can get guaranteed and reliable LNG supply. Therefore, most of the LNG trades are long-term contracts of more than 20 years and the actual price adjusts according to the crude oil price with a floor and ceiling (Zhang and Pang, 2005).

GTL plants are capable of producing a slate of products with highly desirable properties, including lube basestocks, diesels/kerosene, petrochemical naphtha and waxes as Fig. 2 shows. These products meet or exceed virtually all product requirements and, therefore, are fully compatible with petroleum-derived products. F-T diesel is characterized by low sulfur $(\sim 3 \mathrm{ppm})$, low aromatics $(\sim 1 \%)$, a high cetane number $(\sim 70)$, and excellent cold flow properties (Cold Filter Plugging Point, CFPP $<-10{ }^{\circ} \mathrm{C}$ ). These properties make GTL diesel significantly different from diesel derived from crude oil, which is under increasing environmental pressure to reduce its sulfur, nitrogen, olefins, aromatics and metals content. GTL naphtha due to its high paraffin content is an excellent feedstock for petrochemical plants. These environmental benefits of the GTL products make the GTL technology important for the supply of low sulfur, low aromatic transportation fuels (Yang and Wang, 2005; Fleisch et al, 2002; Heng and Idrus, 2004; Bakkerud, 2005; Sie, 1998).

The primary market for GTL products is the everincreasing transportation fuels sector. The current world demand for diesel derived from crude refining is enormous at around $28 \mathrm{MMbpd}\left(1 \mathrm{MMbpd}=5 \times 10^{7} \mathrm{t} / \mathrm{a}\right)$. GTL is considered a very small player in this vast diesel market and such market potential for GTL products can essentially be considered unlimited. The high-quality of GTL diesel exceeds all anticipated future diesel requirement anywhere in the world. More important, the GTL fuels work well in existing infrastructure and in standard diesel fuel engine technology. This is not usually the case for many other alternative fuels that require customized vehicle modifications. A smooth transition can significantly increase the speed of introducing GTL as an alternative fuel into the market. Given this market potential and superior product quality, it is perhaps only a matter of time before F-T-GTL becomes a formidable industry (Patel, 2005).

Unlike LNG, GTL products are commodities that do not require long-term purchase agreements and can be sold in the open market. Although GTL diesel is environmentally superior to diesel derived from crude oil, the pricing mechanism for the GTL products will essentially be similar to that of the refined products, which is essentially benchmarked on crude oil prices (Patel, 2005; Yao, 2005).

\section{Capital costs}

The total capital cost for a typical full chain LNG facility processing 1 BSCFD $\left(103.36 \times 10^{8} \mathrm{~m}^{3} / \mathrm{a}\right)$ is estimated at around 2.4 billion US dollars, which can be conveniently divided into three units: liquefaction facilities (gas plant, liquefaction process, utilities, and offsites), transportation (mainly the LNG ships), and receiving regasification terminals. Among them, the cost for the liquefaction plant is about $52 \%$ of the total cost, the cost for the receiving regasification terminals is about $16 \%$, and the LNG ships is about $32 \%$. During the past ten years, the typical cost for liquefaction has decreased by $25 \%-35 \%$; the cost for transportation has decreased by $20 \%-30 \%$, whereas the decrease of the cost for regasification is much slower. The Fig. 4 is the typical capital cost breakdown of a full chain LNG processing 1 BSCFD (Smith, 2004; Patel, 2005).

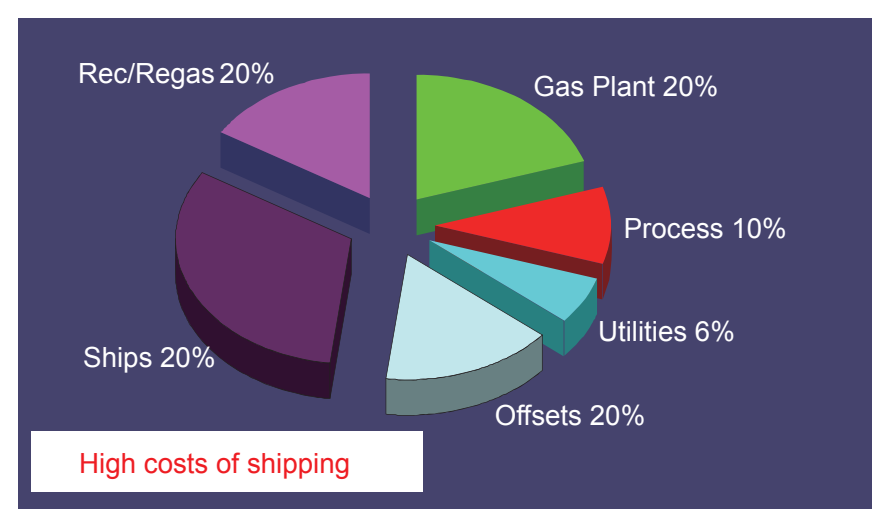

Fig. 4 The typical capital cost breakdown of a LNG facility

Similarly, the capital cost of a GTL processing 1 BSCFD is estimated at around $\$ 2.5$ billion, which can be divided into the following units: gas plant, syngas unit including the air separation unit, Fischer-Tropsch unit, product upgrading unit, other processing units, utilities, and offsites. Significant developments in GTL technology have been made in the past several years and are still ongoing and it very likely to see a continuous downtrend of the overall capital cost of GTL in the near future. Fig. 5 is the typical capital cost breakdown of a full chain GTL processing 1 BSCFD (Smith, 2004; Patel, 2005). 


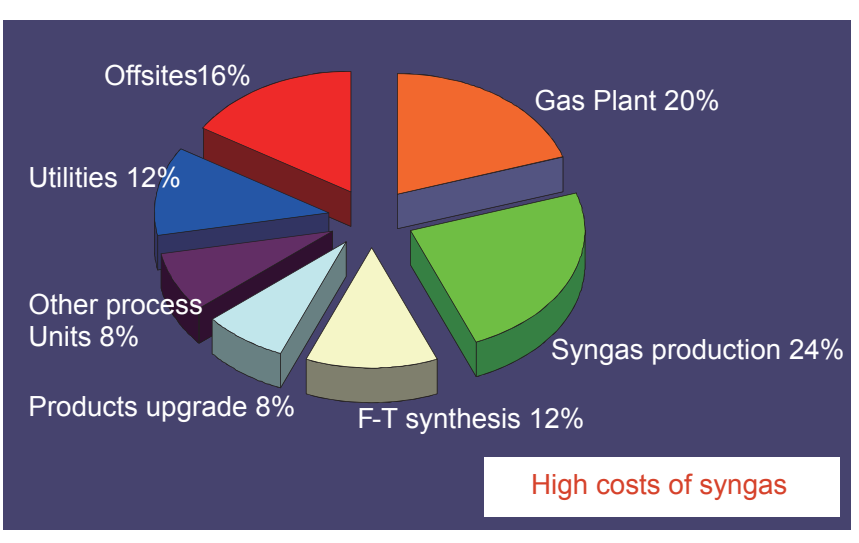

Fig. 5 The typical capital cost breakdown of a GTL facility

Both GTL and LNG require large capital investment, and the magnitude of capital investment is similar for GTL and a full chain LNG facility processing equal amount of natural gas feed. However, the capital costs for LNG production facilities alone are much less than those for GTL because LNG importers usually take the responsibility for investing LNG ships and regasification terminals. For LNG and GTL facilities, the bigger the scale, the more the profit. However, several companies such as Syntroleum and ExxonMobil have developed small-scale GTL technology that requires relatively smaller capital investment. Therefore, the GTL production is more flexible and can easily be regulated according to the marketing and international circumstances, making GTL more suitable for small "stranded" natural gas storages (Hu et al, 2006; Yao, 2005; Han et al, 2006; Antari and Mokrani, 2002; Qian and Zhu, 2007 ).

\section{Economic evaluation}

The economic evaluation of GTL versus LNG can be conducted in terms of production costs and product value (Patel, 2005). Table 1 presents the costs of producing GTL transportation fuels and LNG at a natural gas price of $\$ 7.0-11.0 / \mathrm{MMBTU}$ and refinery fuels at a crude oil price of $\$ 80-120 / \mathrm{bbl}(1 \mathrm{bbl}=0.14$ tonnes). Since one barrel of GTL product requires approximately $10 \mathrm{MMBTU}$ of natural gas, the comparison of the production costs in Table 1 is made at a standard of 10 MMBTU natural gas feedstocks. Although the operating and capital costs are higher for GTL than for refinery products, the overall cost of producing diesel from gas by GTL is similar with that from crude oil by refinery. The cost of LNG production corresponding to $10 \mathrm{MMBTU}$ of natural gas is estimated to be $\$ 80-125$, which is slightly smaller but close to that of GTL. Therefore, the profitability of the two gas monetizing options is essentially governed by the final value of the products.

Table 2 compared the production value (revenue) generated from the same quantity of gas (10 MMBTU) between LNG and GTL. If the typical current market price of the GTL diesel is assumed as $\$ 120-160 / \mathrm{bbl}$ and one barrel of GTL product requires approximately $10 \mathrm{MMBTU}$ of natural

Table 1 Production cost of GTL transportation fuel, Refinery fuel and LNG

\begin{tabular}{c|cccc}
\hline \multirow{4}{*}{ Cash costs } & Facility & GTL & Refinery & LNG \\
\cline { 2 - 5 } & Natural gas (10 MMBTU) & $\$ 70-110$ & -- & $\$ 70-110$ \\
\cline { 2 - 5 } & Crude oil (1 Barrel) & -- & $\$ 80-120$ & -- \\
\hline \multirow{2}{*}{ Operating costs } & $\$ 6-8$ & $\$ 2-3$ & $\$ 2-3$ \\
\hline & Capital costs & $\$ 9-14$ & $\$ 4-7$ & $\$ 8-12$ \\
\hline
\end{tabular}

Table 2 Production value of GTL and LNG

\begin{tabular}{ccc}
\hline Facility & GTL & LNG \\
\hline Market price of product & $\$ 120-160 / \mathrm{bbl}$ & $\$ 14.0-18.0 / \mathrm{MMBTU}$ \\
Conversion efficiency & $60 \%$ & $88 \%$ \\
Feed & $10 \mathrm{MMBTU}$ & $1.14 \mathrm{MMBTU}$ \\
Production value of feed & $\$ 12.0-16.0$ & $\$ 12.32-15.84$ \\
\hline
\end{tabular}


gas, the ultimate production value of the natural gas resource is \$12.0-16.0 per MMBTU for GTL. If the typical current market price of LNG is \$14.0-18.0/MMBTU, taking account of the conversion efficiency, the ultimate production value of the natural gas resource can be calculated as \$12.32-15.84 per MMBTU for LNG. As the production cost, the product value of the natural gas resource appears to be very similar based on the above pricing assumptions for the two systems.

The above analysis suggests comparable investments for both GTL and LNG. The long term pricing mechanism for LNG is not conducive to maximize the resource revenue. The GTL product value on the other hand is vulnerable to crude oil prices. However, due to the high quality of GTL products, whose value is at the uptrend, under the 'normal' crude oil pricing range GTL appears to offer better revenue for the resources (Smith, 2004; Yao, 2005; Kashav and Basu, 2007). Table 3 is the economic analysis of GTL and LNG by Syntroleum Company for the gas resources at Yamal peninsula, which forecasts similar investment and a fairly higher profitability of GTL for large-scale natural gas reservoirs (Qian and Zhu, 2007). Although the analysis of Table 3 is made in 2004 with the price for crude oil and natural gas increasing significantly during the past several years, the forecast still works reasonably due to the similarscale increase of crude oil and natural gas price.

Table 3 Economic analysis of GTL and LNG for large-scale natural gas reservoirs

\begin{tabular}{ccc}
\hline & GTL & LNG \\
Product price & $\$ 220 / \mathrm{t}$ & $\$ 140 / \mathrm{t}$ \\
Sale volume & $550 \times 10^{4} \mathrm{t}$ & $640 \times 10^{4} \mathrm{t}$ \\
Facility investment & $\$ 3.4$ billion & $\$ 2.3$ billion \\
Oil tanker investment & $\$ 0.3$ billion & $\$ 1.0$ billion \\
Total investment & $\$ 3.7$ billion & $\$ 3.3$ billion \\
Market location & Rotterdam & Zeebrugge \\
Market distance & $4000 \mathrm{~km}$ & $4000 \mathrm{~km}$ \\
Internal repayment rate & $15 \%$ & $12 \%$ \\
\hline
\end{tabular}

Notes: Data from Petroleum Review, 2004, 58(690): 14-16

\section{Summary and perspective}

In summary, GTL F-T technology is beginning to show commercial viability, whereas LNG has been well established. A GTL facility is more complex, has lower plant efficiency and is more expensive than an LNG facility. However the full chain capital expenses of both GTL and LNG are comparable. Due to the similar capital investment the decision to invest in LNG or GTL from a resource owner' $\mathrm{s}$ perspective can be challenging. Besides the capital, other factors, such as technology risks, plant availability, local market, overall company strategy and political consideration are also important in the decision-making.

GTL and LNG serve different energy markets and both are attractive for monetization of stranded gas reserves. GTL products, dependent upon the crude oil price, exhibit slightly higher value per MMBTU than LNG. Technological improvement and compelling investment from the world's major oil companies suggest that the GTL industry is likely to expand rapidly over the next decade and will develop into a significant commercial factor in world energy markets over the next few years. More GTL means that less LNG will be available on the world market, slowing the development of competition and resulting in higher prices and less available supply of LNG, potentially altering LNG's projected role in the world's natural gas market.

\section{References}

Antari A, Mokrani T. Gas to liquids technology-A new approach for marketing natural gas. 17th World Petroleum Congress, Rio De Janeiro, 2002. Block 3/Forum 18/Paper 12

Bakkerud P K. Update on synthesis gas production for GTL. Catalysis Today. 2005. 106: 30-33

Chen Z. The 17th World Petroleum Congress' view on natural gas development and utilization. Petroleum Processing and Petrochemicals. 2003. 34(4): 1-6 (in Chinese) 
Fleisch T H, Sills R A and Briscoe M D. Emergence of the gas-to-liquids industry: A review of global GTL developments. Journal of Natural Gas Chemistry. 2002. 11: 1-14

Han D, Pan J and Jiang J. Progress of making synthetic oil from natural gas and its technico-economic analysis, Chemical Technology Market. 2006. 29(6): 27-41 (in Chinese)

Heng $\mathrm{H} \mathrm{C}$ and Idrus $\mathrm{S}$. The future of gas to liquids as a gas monetisation option. Journal of Natural Gas Chemistry. 2004. 13: 63-70

Hu J, Zhu B and Wang J. Natural gas chemical technology and application. Beijing: Chemical Industry Press, 2006 (in Chinese)

Kashav T S and Basu S. Gas-to-liquid technologies: India's perspective. Fuel Processing Technology, 2007. 88: 493-500

Patel B. Gas Monetisation: A technico-economic comparison of gasto liquids and LNG. 7th World Congress of Chemical Engineering. Glasgow. 2005

Pyrdol $\mathrm{J}$ and Baron B. What is more profitable to build: GTL production facilities or LNG liquefaction plants. Presented at World Natural Gas Market and Trade (EMF-23), Berlin Meeting. June 2006.

Qian B and Zhu J. Status and development trend of natural gas compound. Natural Gas and Oil. 2007. 25(4): 23-28 (in Chinese)

Sie S T. Process development and scale up: IV case history of the development of a Fischer-Tropsch synthesis process. Reviews in Chemical Engineering. 1998. 14: 109-157

Smith R M. New developments in gas to liquids technology. Presented at CERI 2004 Petrochemical Conference. Delta Lodge at Kananaskis, Alberta, Canada

Wang Y. The opportunity, risk, and challenge in LNG industry. International Petroleum Economics. 2005. 13(6): 39-43 (in Chinese)

Yang G and Wang X. Liquefaction technology of natural gas. Natural Gas and Oil. 2005. 23(2): 10-16 (in Chinese)

Yao G. Development of GTL and related technico-economic analysis, International Petroleum Economics. 2005. 13(5): $23-29$ (in Chinese)

Zeng $\mathrm{H}$. Trends of development in the global LNG market. International Petroleum Economics, 2006. 14(6): 53-56 (in Chinese)

Zhang K and Pang M. The present and future of the world's LNG industry. International Petroleum Economics. 2005. 13(10): $55-59$ (in Chinese)

(Edited by Zhu Xiuqin) 